\title{
Precoding of Correlated Symbols for STBC Systems Design
}

\author{
Kelvin Anoh ${ }^{1}$, Bamidele Adebisi ${ }^{1}$ and Godfrey Okorafor ${ }^{2}$ \\ ${ }^{1}$ School of Engineering, Manchester Metropolitan University, UK \\ ${ }^{2}$ Novena University, Nigeria \\ k.anoh@mmu.ac.uk, b.adebisi@mmu.ac.uk, nwajigo@novenauniversity.edu.ng
}

\begin{abstract}
A problem with transmitting correlated symbols over multiple transmit channel paths is that there is no diversity gain achieved at the receiver. Precoding technique provides a smart approach to achieving diversity gains at the receiver even when correlated symbols are transmitted; by phase variation, amplitude variation or both provided by the precoder. The space-time block code (STBC) technique, for example, is well-known when transmitting the same symbols by making them appear as different symbols using conjugation. We observe that correlated symbols can be transmitted over multiple transmit channel paths over STBC scheme while still achieving diversity. The correlated symbols can be made to appear as different symbols by using precoders; this enables diversity and improves data rate. Combining the proposed with the equivalent channel matrix (EVCM) permits the proposed design to outperform the conventional precoding of uncorrelated symbols technique by $2 \mathrm{~dB}$ at all bit error ratio (BER) for and antenna configurations. This is useful in increasing data rates with better BER performance.
\end{abstract}

Key words: STBC, Correlated, MIMO, Precoding

\section{Introduction}

Since the advent of $4 \mathrm{G}$ technology, a lot of work has been done towards achieving a $5 \mathrm{G}$ design $[1,2,3]$. Most of these works have been done on beamforming especially for mmWave and $5 \mathrm{G}[3,4]$. Beamforming is a precoding technique that involves weighting some input symbols to enable some transmissions in the desired directions or to specific users [5]. The beamforming precoding technique has been investigated widely for STBCs $[6,7,8]$. In the use of STBC to design multi-antenna systems, the scheme enables that the same symbol is sent to the receiver more than once by making that same symbol look like another 
one; by conjugation. To transfer decoding complexity from the receiver to the transmitter, an equivalent channel matrix (EVCM) is usually derived. This technique also enables linear processing and simplifies receiver design. With the EVCM, the implementation of STBC reduces to sending $N_{T}$-unique symbols to the receiver over the channel (EVCM) matrix. When combined with precoding, the preocder weights are multiplied by these symbols and equivalently received in the receiver. Conventional transmit precoding, for instance angular beamforming differentiates among these symbols by assigning different phases to each transmit symbol block [9]. If these possibilities and differentiations already exist among the symbols (e.g. EVCM and phase difference), we consider that these differences already provided enough diversity for transmitting different symbols than transmitting the same symbols as different symbols over many timeslots as in [10]. Consequently, suppose that the $N_{T}$-transmit symbols are correlated. One, the data rate is increased, the amplitude of the symbols are also amplified accordingly but not reduced to $K<L$ symbol lengths like when using the conventional STBC technique; $K=\left(L / N_{T}\right)$. We investigate these in this paper for standard orthogonal-STBC (OSTBC) with two transmit antennas $\left(N_{T}=2\right)$.

In Section 2, we present the system model and discuss the method of multiantenna precoding that we propose in Section 3. The simulation method and results are described in Section 4 with the conclusions following.

\section{System Model}

In our design, we consider an STBC system equipped with two transmit antennas $\left(N_{T}=2\right)$ and some $N_{R}$ receivers. Since there are $N_{T}$ transmitting branches and $N_{R}$ receivers, we summarize the $\left(N_{R} \times N_{T}\right)$ MIMO system in linear form as follows

$$
\overline{\mathcal{Y}}=\overline{\mathcal{H}} \overline{\mathcal{C}}+\overline{\mathcal{Z}}
$$

where $\overline{\mathcal{Y}}$ is the received signal at the receiver, $\overline{\mathcal{H}} \in \mathbb{C}^{N_{R} \times N_{T}}$ is a flat fading multipath channel and $\overline{\mathcal{Z}} \sim \mathbb{C N}\left(0, \sigma_{z}^{2} \boldsymbol{I}_{N_{R}}\right)$ is the additive white Gaussian noise with zero mean and variance $\sigma_{z}^{2}$. The received signal $\overline{\mathcal{Y}}$ is $\overline{\mathcal{Y}} \in \mathbb{C}^{N_{R} \times L}$. The multispatial data, $\overline{\mathcal{C}} \epsilon \mathbb{C}^{N_{T} \times L}$ is formed from weighting some un-precoded phase-shift keying (PSK) input symbols. These weights of the multi-spatial data constitute the precoders and can be discussed as

$$
\overline{\mathcal{C}}=\boldsymbol{w} \times \overline{\boldsymbol{c}}
$$

where $\boldsymbol{w} \epsilon \mathbb{C}^{N_{T} \times 1}$ is the transmit precoder and $\overline{\boldsymbol{c}} \in \mathbb{C}^{1 \times L}$ is the un-precoded PSK symbols. Since the precoder enables the realization of multi-spatial data streams in (2), then one can easily rewrite (1) as

$$
\overline{\mathcal{Y}}=\overline{\mathcal{H}} \sum_{i=1}^{N_{T}}\left(w_{i} \overline{\boldsymbol{c}}_{i}\right)+\mathcal{Z}
$$


Each of the $\overline{\boldsymbol{c}}_{i} \forall i=1, \cdots, N_{T}$ constitutes the $i^{\text {th }}$ transmitting branch symbol corresponding to the $i^{\text {th }}$ precoder. Let the un-precoded symbols be the standard STBC code described in [10], we rewrite $\overline{\boldsymbol{c}}$ as

$$
\check{\mathcal{C}}=\left[\begin{array}{cc}
\boldsymbol{c}_{1} & \boldsymbol{c}_{2} \\
-\boldsymbol{c}_{2}^{*} & \boldsymbol{c}_{1}^{*}
\end{array}\right]
$$

where $(\cdot)^{*}$ represents complex conjugate. Herein (4), each of the $\boldsymbol{c}_{i} \forall i=1, \cdots, N_{T}$ constitutes the $i^{\text {th }}$ transmitting timeslot symbol of the OSTBC Alamouti code. It can easily be verified that (4) enables orthogonal processing such as $|\check{\mathcal{C}}|^{2}=$ $\operatorname{diag}\left(\left|\boldsymbol{c}_{1}\right|^{2}+\left|\boldsymbol{c}_{2}\right|^{2},\left|\boldsymbol{c}_{1}\right|^{2}+\left|\boldsymbol{c}_{2}\right|^{2}\right.$ ). Using (4) an EVCM can be derived so that the linear system in (1) can be rewritten as

$$
\mathcal{Y}=\mathcal{H C}+\mathcal{Z}
$$

where $\mathcal{H}=\left[\mathcal{H}_{1} \mid \mathcal{H}_{2}\right]$ represents the channel states at two different timeslots and $\mathcal{C}=\boldsymbol{w} \times \boldsymbol{c} ; \boldsymbol{c}_{i}(l) \in \mathbb{C}^{N_{T} \times L} \forall l=1, \cdots, L$. We showed in [11] that an equivalent channel matrix can be derived to simplify detection in the receiver as

$$
\mathcal{H}=\left[\begin{array}{cc}
\boldsymbol{h}_{1} & \boldsymbol{h}_{2} \\
\boldsymbol{h}_{2}^{*} & -\boldsymbol{h}_{1}^{*}
\end{array}\right]
$$

Both (4) and (6) are used for $N_{T}=2$ and $N_{R}=1$ designs. We described designs that enable (6) to be used for MIMO system in [11]. Using (6) in (5) for a conventional STBC [10], $\mathcal{C}_{\epsilon} \mathbb{C}^{N_{T} \times K}$ and $\forall N_{T} \mathcal{C}_{i} \neq \mathcal{C}_{j}$ because $\boldsymbol{c}_{i} \neq \boldsymbol{c}_{j} \forall i=$ $1, \cdots, N_{T}$ and $\boldsymbol{c}_{i, j}(k) \in \mathbb{C}^{1 \times K} \forall k=1, \cdots, K ; K=\left(L / N_{T}\right)$. Note that $\forall N_{T}, i \neq j$. Since there are $N_{T}$ separate beams sequel to the precoder $(\boldsymbol{w})$ each equipped with a unique phase angle and amplitude, we consider the case where $\forall i=$ $1, \cdots, N_{T} \boldsymbol{c}_{i}=\boldsymbol{c}_{j}$ where $\boldsymbol{c}_{i, j}(l) \epsilon \mathbb{C}^{1 \times L} \forall l=1, \cdots, L ; \forall N_{T}, i \neq j$. We argue that since the precoder has uniquely different phases and amplitudes, then $\forall N_{T} \mathcal{C}_{i} \neq$ $\mathcal{C}_{j}$ still holds thus achieving diversity that does not exist when only $\boldsymbol{c}_{i}=\boldsymbol{c}_{j}, \forall i=$ $1, \cdots, N_{T}$ is used. We maintain that although $\boldsymbol{\mathcal { R }}_{c_{i} c_{j}}=\sum\left(c_{i}-\mu_{c_{i}}\right)\left(c_{j}-\mu_{c_{j}}\right)=$ $\boldsymbol{I}$, when the precoder is applied, then $\boldsymbol{\mathcal { R }}_{\mathcal{C}_{i} \mathcal{C}_{j}}=\sum\left(\mathcal{C}_{i}-\mu_{\mathcal{C}_{i}}\right)\left(\mathcal{C}_{j}-\mu_{\mathcal{C}_{j}}\right) \neq \boldsymbol{I}$; $\mu_{x}=\int x p(x) d x$ is the statistical mean and $\boldsymbol{I}$ is an identity matrix. Moreover, since $K<L$, then the proposed scheme provides higher data rate advantage with the amplitude improved so that the BER becomes better than the traditional STBC combined with the precoding scheme.

\section{Method of Multi-antenna Precoding}

Singular value decomposition (SVD) provides an elegant method for realizing well-performing precoders [5]. For instance, the precoder weights are unitary matrices that are also positive definitive. When used with the channel matrix, the SVD provides unitary matrices (or the precoders) that simplify channel compensation at the receiver. As an example, consider the EVCM channel matrix, $\mathcal{H}$, its SVD can be expressed as $[12,13,14]$ 


$$
\mathcal{H}=\mathcal{U} \Omega \mathcal{V}^{\mathcal{H}}
$$

where $\mathcal{U}$ and $\mathcal{V}$ are unitary matrices of the $N_{T} \times N_{T}$ dimension of the transmitter and $N_{R} \times N_{R}$ dimension of the receiver respectively. It is easy to verify that $\mathcal{U}^{\mathcal{H}} \mathcal{U}=\boldsymbol{I}_{N_{R} \times N_{R}}$ and also $\mathcal{V}^{\mathcal{H}} \mathcal{V}=\boldsymbol{I}_{N_{T} \times N_{T}}$. Given the EVCM in (6), $\mathcal{H}$ is a square matrix. Then, the diagonal matrix $\Omega \in \mathbb{R}^{N_{T} \times N_{T}}$ corresponds to the power allocated to each of the $N_{T}$ channels. The input signal $(\mathcal{C})$ can also be represented, following (3), as

$$
\mathcal{C}=\mathcal{V} \times\left(\boldsymbol{I}_{N_{T}} \otimes \boldsymbol{c}\right)
$$

where $\otimes$ is the Kronecker product operator, $\mathcal{C} \in \mathbb{C}^{N_{T} \times L}$ and $\mathcal{V}^{\mathcal{H}}$ is a vector of the eigen-decomposition of the channel $\mathcal{H} \epsilon \mathbb{C}^{N_{R} \times N_{T}}$ for a flat fading channel model. At the receiver, the detection of the transmitted symbols can be explored that by using (7) in (5)

$$
\begin{aligned}
\hat{\boldsymbol{c}} & =\mathcal{U}^{\mathcal{H}} \mathcal{Y} \\
& =\mathcal{U}^{\mathcal{H}} \mathcal{H C}+\mathcal{U}^{\mathcal{H}} \mathcal{Z} \\
& =\mathcal{U}^{H}\left(\mathcal{U} \Omega \mathcal{V}^{\mathcal{H}}\right) \mathcal{C}+\mathcal{U}^{\mathcal{H}} \mathcal{Z} \\
& =\mathcal{U}^{\mathcal{H}}\left(\mathcal{U} \Omega \mathcal{V}^{\mathcal{H}}\right) \mathcal{V} \boldsymbol{c}+\mathcal{U}^{\mathcal{H}} \mathcal{Z} \\
& =\boldsymbol{\Omega} \boldsymbol{c}+\mathcal{U}^{\mathcal{H}} \mathcal{Z}
\end{aligned}
$$

The result provides $N_{T}$ parallel independent subchannels. Suppose that $\mathcal{V}^{\mathcal{H}}$ is used at the transmitter for precoding the input symbols, it is clear that $\mathcal{V}^{\mathcal{H}}$ provides different phase and amplitudes for each transmitting branch. Thus, if the input symbols are correlated, then precoding each symbol with $\mathcal{V}^{\mathcal{H}}$ provides uncorrelation among these symbols. Then, if we rewrite $\boldsymbol{w}_{t}=\mathcal{V}^{\mathcal{H}}$ as the precoder at the transmitter and $\boldsymbol{w}_{r}^{\mathcal{H}}=\mathcal{U}$ for compensation at the receiver, the received signal can be expressed as

$$
\begin{aligned}
\hat{\boldsymbol{c}} & =\boldsymbol{w}_{r}^{\mathcal{H}} \mathcal{Y} \\
& =\boldsymbol{w}_{r}^{\mathcal{H}} \mathcal{H C}+\boldsymbol{w}_{r}^{\mathcal{H}} \mathcal{Z} \\
& =\boldsymbol{w}_{r}^{\mathcal{H}} \mathcal{H} \boldsymbol{w}_{t} \boldsymbol{c}+\boldsymbol{w}_{r}^{\mathcal{H}} \mathcal{Z}
\end{aligned}
$$

On the other hand, since $\boldsymbol{w}_{r}^{\mathcal{H}}$ is a unitary matrix, the estimated noise term $\hat{\mathcal{Z}}=\left(\boldsymbol{w}_{r}^{\mathcal{H}} \mathcal{Z}\right)$ remains Gaussian. For MIMO systems, if the detecting matrix $\boldsymbol{w}_{r}^{\mathcal{H}}$ maximizes the objective function $\left|\boldsymbol{w}_{r}^{\mathcal{H}} \mathcal{H} \boldsymbol{w}_{t}\right|$ at the receiver given $\boldsymbol{w}_{t}$, then the receiver is said to attain maximum ratio combining (MRC) [15]. Clearly, given the objective function $\left|\boldsymbol{w}_{r}^{\mathcal{H}} \mathcal{H} \boldsymbol{w}_{\boldsymbol{t}}\right|$, the detecting matrix $\boldsymbol{w}_{r}^{\mathcal{H}}$ enables the receiver to achieve maximal output for $N_{T}=2$. This may not be necessarily true for higher order designs of STBC systems except when the scheme has been enabled to achieve full-diversity such as in $[16,17,18,19]$. 
The SNR at the receiver can be well-described as

$$
\gamma=\frac{\mathbb{E}\left\{\left|\boldsymbol{w}_{r}^{\mathcal{H}} \mathcal{H} \boldsymbol{w}_{\boldsymbol{t}} \boldsymbol{c}\right|^{2}\right\}}{\mathbb{E}\left\{\left|\boldsymbol{w}_{r}^{\mathcal{H}} \mathcal{Z} \mathcal{Z}^{\mathcal{H}} \boldsymbol{w}_{r}\right|\right\}}=\mathbb{E}\left\{\left|\boldsymbol{w}_{r}^{\mathcal{H}} \mathcal{H} \boldsymbol{w}_{\boldsymbol{t}}\right|^{2}\right\} \frac{E_{c}}{\sigma_{\bar{z}}^{2}}
$$

where $E_{c}=\mathbb{E}\left\{|\boldsymbol{c}|^{2}\right\}$ and $\sigma_{\bar{z}}^{2}=\mathbb{E}\left\{\left|\mathcal{Z} \mathcal{Z}^{\mathcal{H}}\right|\right\} ; \mathbb{E}\{\cdot\}$ is the statistical expectation mean of the precoded symbols at the receiver. For MIMO designs, the exact $\mathrm{SNR}$ at the receiver can be expressed as $\gamma_{M I M O}=\sum_{j=1}^{N_{R}} \sum_{i=1}^{N_{T}} \gamma_{i, j}$. Considering $M$-ary PSK symbols, the error probability can be described as [6]

$$
P_{p s k}=\frac{1}{\pi} \int_{0}^{(M-1) \pi / M} \prod_{i=1}^{N_{T}} \xi_{i}\left(\gamma_{i}, b_{p s k}, \theta\right) d \theta
$$

where $\theta=\pi / 2, \xi_{i}\left(\gamma_{i}, b_{p s k}, \theta\right)=-b_{p s k} \gamma / \sin ^{2} \theta$ and $b_{p s k}=\sin ^{2}(\pi / M)$. Define the original un-precoded PSK symbol, $\mathbf{s}=\mathbb{C}^{1 \times L}$ where $L$ is the length of the input symbols. For multiple antennas dispensing with correlated symbols $\mathcal{C}(l) \epsilon \mathbb{C}^{N_{T} \times L} ; \mathcal{C}=\mathcal{V} \times\left(\boldsymbol{I}_{N_{T}} \otimes \boldsymbol{c}\right)$. For conventional STBC symbols, $\mathcal{C}(k) \epsilon \mathbb{C}^{N_{T} \times K}$ where $K<L$ and $K=L / N_{T} ; k=1, \cdots, K$. For the precoded conventional STBC design equipped with $N_{T}=2$ and $N_{R}=1$, the SNR is as defined in (11). On the other hand, for correlated symbols similarly equipped, then

$$
\gamma_{\text {corr }}=\frac{N_{T} \mathbb{E}\left\{\left|\boldsymbol{w}_{r}^{\mathcal{H}} \mathcal{H} \boldsymbol{w}_{\boldsymbol{t}} \boldsymbol{c}\right|^{2}\right\}}{\mathbb{E}\left\{\left|\boldsymbol{w}_{r}^{\mathcal{H}} \mathcal{Z} \mathcal{Z}^{\mathcal{H}} \boldsymbol{w}_{r}\right|\right\}}=N_{T} \mathbb{E}\left\{\left|\boldsymbol{w}_{r}^{\mathcal{H}} \mathcal{H} \boldsymbol{w}_{\boldsymbol{t}}\right|^{2}\right\} \frac{E_{c}}{\sigma_{\bar{z}}^{2}}
$$

Comparing (11) and (12), it can be observed that the received SNR for the correlated symbols is better than the uncorrelated symbols by $N_{T}$. If the system operates with $N_{R}>1$, then $\mathcal{C}(k) \in \mathbb{C}^{N_{R} N_{T} \times \frac{L}{N_{T}}}$ for the uncorrelated and the SNR is

$$
\gamma_{\text {uncorr }, M I M O}=\sum_{n=1}^{N_{R}} \sum_{i=1}^{N_{T}} \gamma_{i, n}
$$

while $\mathcal{C}(l) \epsilon \mathbb{C}^{N_{R} N_{T} \times L}$ so that the SNR for the correlated input symbols becomes

$$
\gamma_{c o r r}=N_{T} \gamma_{\text {uncorr }, \text { MIMO }}=\sum_{n=1}^{N_{R}} \sum_{i=1}^{N_{T}}\left(N_{T} \gamma_{i, n}\right)
$$

The above results show that the SNR increases with increasing both the number of transmitting antennas and the number of receivers. If the receiver is constrained in size, for example mobile phones, tablets or laptops in such a way that the integration of many multiple receiving antennas is problematic due to mutual coupling, then the number of transmitting antennas can be increased to improve throughput at the receiver. These results are demonstrated using simulation described in Section 4 . 


\section{Simulation Results and Discussions}

The simulation environment involves a randomly generated input symbols of length $L$ demultiplexed into $N_{T}$ branches to realize some $N_{T}$ uncorrelated symbols; this reduces the symbol length for each branch to $K=\left(L / N_{T}\right)$ such that $K<L$. Thus, the $i^{t h}$-branch input symbol constitutes a symbol vector $\boldsymbol{c}_{i} \in \mathbb{C}^{1 \times \frac{L}{N_{T}}} \forall i=1, \cdots, N_{T}$ and $\boldsymbol{c}_{i} \neq \boldsymbol{c}_{j}$ for all transmitting branches; this enables the standard STBC symbols that are uncorrelated. A multipath flat fading channel with $\mathcal{H} \sim \mathcal{C N}\left(0, \sigma_{h}^{2} \boldsymbol{I}\right)$ distribution was generated and used to construct an EVCM. With the EVCM only the $\boldsymbol{c}_{i} \epsilon \mathbb{C}^{1 \times K} \forall i=1, \cdots, N_{T}$ uncorrelated symbols are used and these are precoded before combining them with the EVCM channel. On the other hand, since the precoding involves different weights of different amplitudes and phases, the default input symbols of $L$ length are used when realizing the correlated symbols. This fact enables that the gain in the receiver can be increased by $N_{T}$. As a consequence, this technique doubles the data rate against the uncorrelated design for $N_{T}=2$ and improves the processing time since the multiple conjugation operations to recover the original symbols are not required. The receiver involves only a linear processing which further makes the design elegant.

Using simulation we show that with the precoding, multiplying the received signal $\boldsymbol{Y}$ with $\boldsymbol{w}_{r}^{\mathcal{H}}$ is equivalently a diagonal matrix as the conventional channel compensation if the channel matrix can be derived; meanwhile only $\boldsymbol{w}_{r}^{\mathcal{H}} \mathcal{H} \boldsymbol{w}_{t}=$ $\left(\mathcal{H}^{\frac{1}{2}}\right)^{\mathcal{H}} \mathcal{H}^{\frac{1}{2}}$. In Fig. 1 the results for transmitting correlated and uncorrelated precoded symbols are compared. Alongside, the result of default OSTBC is presented also. Since $\boldsymbol{w}_{r}^{\mathcal{H}} \mathcal{H} \boldsymbol{w}_{t}$ is only equivalent to $\left(\mathcal{H}^{\frac{1}{2}}\right)^{\mathcal{H}} \mathcal{H}^{\frac{1}{2}}$, then the received SNR of the default STBC will slightly better than that of the uncorrelated precoded design and correspondingly their BER. These are achieved in Figs. 1 and 2.

First, observe that our results in Fig. 1 are consistent with the ones reported in [10] for $2 \times 1$ and $2 \times 2$ antenna configurations. Comparing the proposed design with the standard OSTBC for 2 antennas with one receiver, it can be seen that the correlated symbol consistently achieved $2 \mathrm{~dB}$ performance gain over the standard OSTBC equipped with one receiver. In (12) and (13), we showed that the received SNR is a function of both $N_{T}$ and $N_{R}$. Consequently, comparing the designs for $2 \times 2$ antenna configurations, the correlated symbol precoding technique achieves $2 \mathrm{~dB}$ better than the uncorrleated symbols. These investigations are limited to BPSK modulation. Next, we investigate these designs for higher spectral efficiency using QPSK modulation in Fig. 2.

With a similar design environment as in 1 for a QPSK design in Fig. 2, it can be seen that the proposed consistently outperformed the default-OSTBC by $2 \mathrm{~dB}$ for both $2 \times 1$ and $2 \times 2$ antenna configurations. If the EVCM is considered in terms of the standard STBC matrix, there are 2 timeslots and 2 antenna spaces so that the system attains full spatial rate (and also full diversity since $\boldsymbol{w}_{r}^{\mathcal{H}} \boldsymbol{H} \boldsymbol{w}_{t}=\lambda \boldsymbol{I}_{N_{T}}$ and $\mathcal{H}^{\mathcal{H}} \mathcal{H}=\sigma_{h}^{2} \boldsymbol{I}_{N_{T}}$, where $\lambda$ is the eigenvalue from the SVD decomposition of 


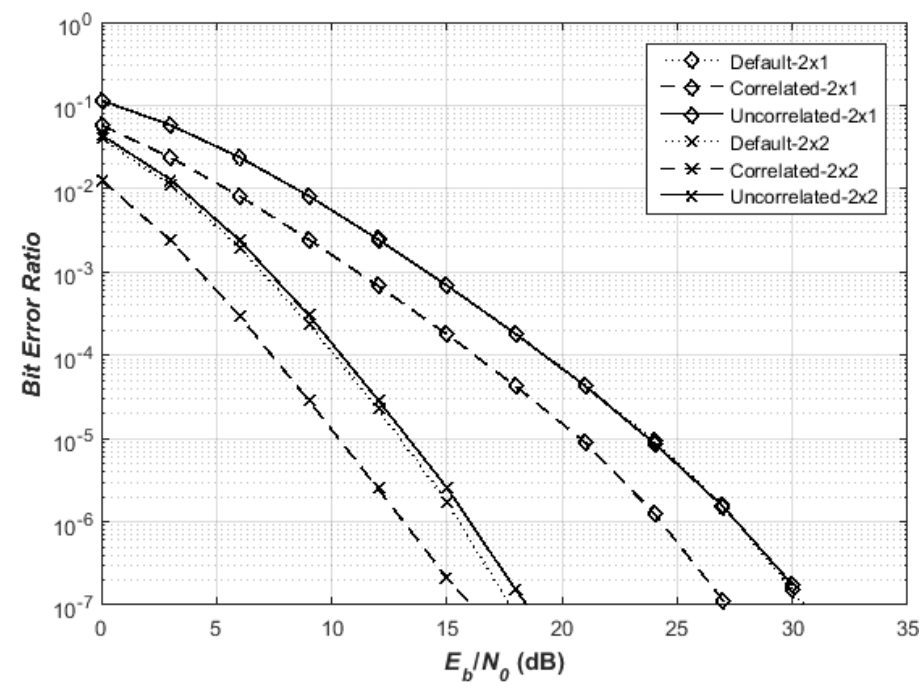

Figure 1: Comparison of Precoded Correlated and uncorrelated symbols for MIMO STBC with 2 Transmit antennas (BPSK)

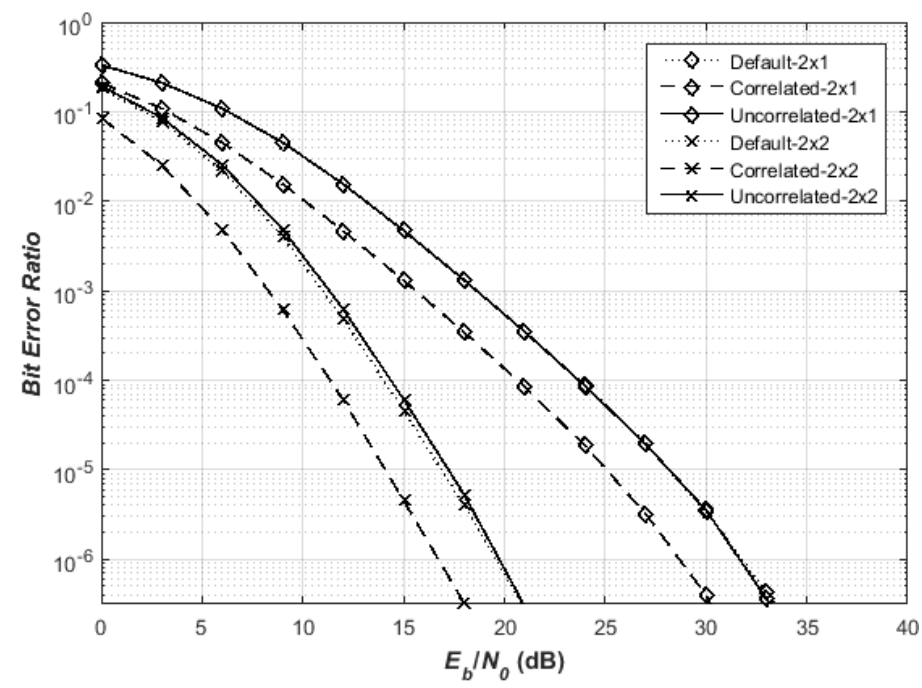

Figure 2: Comparison of Precoded Correlated and uncorrelated symbols for MIMO STBC with 2 Transmit antennas (QPSK) 
the EVCM). In each of the timeslots, more symbols are transmitted with the correlated symbols than in the uncorrelated symbols per timeslot thus achieving a higher data rate. It follows also that in addition to achieving diversity gain, the SNR is improved in the order of transmitting antennas.

\section{Conclusion}

In this paper we have introduced the concept of precoding correlated symbols over STBC scheme. Although corrected symbols sent over many transmitting channels do not provide any diversity at the receiver, we introduced a novel approach to enabling diversity by transmitting precoded symbols over uncorrelated channels. The correlated symbols are made uncorrelated by precoding them before transmission over multipath channels. These precoding weights provide variations in both phases and amplitudes of the symbols so that diversity gain and some coding performance gain are achieved. We found that the data rates of the correlated symbols are $N_{T}$ multiples of those of the uncorrelated symbols over an OSTBC scheme. These translated into $2 \mathrm{~dB}$ gain when we used BPSK and QPSK modulation schemes for $2 \times 1$ and $2 \times 2$ antenna configurations. Thus, although STBC provides a smart method of transmitting the same signals as uncorrelated different signals by conjugation, precoding of correlated signals over an STBC scheme realized through EVCM reduced the error probability by $N_{T}$ and doubles the BER performance of an STBC design. This translates to increased data over the same spectral conditions and lower signal power required to drive more data symbols with improved BER. The proposed has the potential for better performances with higher order antenna configurations.

\section{References}

1. S Mohammad Razavizadeh, Minki Ahn, and Inkyu Lee. Three-dimensional beamforming: A new enabling technology for $5 \mathrm{~g}$ wireless networks. IEEE Signal Processing Magazine, 31(6):94-101, 2014.

2. Tatsunori Obara, Satoshi Suyama, Jiyun Shen, and Yukihiko Okumura. Joint fixed beamforming and eigenmode precoding for super high bit rate massive MIMO systems using higher frequency bands. In IEEE 25th Annual International Symposium on Personal, Indoor, and Mobile Radio Communication (PIMRC), pages 607-611, 2014.

3. Wonil Roh, Ji-Yun Seol, Jeongho Park, Byunghwan Lee, Jaekon Lee, Yungsoo Kim, Jaeweon Cho, Kyungwhoon Cheun, and Farshid Aryanfar. Millimeter-wave beamforming as an enabling technology for $5 \mathrm{~g}$ cellular communications: theoretical feasibility and prototype results. IEEE Communications Magazine, 52(2):106-113, 2014.

4. Ahmed Alkhateeb, Jianhua Mo, Nuria Gonzalez-Prelcic, and Robert W Heath. MIMO precoding and combining solutions for millimeter-wave systems. IEEE Communications Magazine, 52(12):122-131, 2014. 
5. C-C Jay Kuo, Shang-Ho Tsai, Layla Tadjpour, and Yu-Hao Chang. Precoding techniques for digital communication systems. Springer, 2008.

6. Shengli Zhou and Georgios B Giannakis. Optimal transmitter eigenbeamforming and space-time block coding based on channel correlations. IEEE Transactions on Information Theory, 49(7):1673-1690, 2003.

7. Li Liu and Hamid Jafarkhani. Application of quasi-orthogonal space-time block codes in beamforming. IEEE Transactions on Signal Processing, 53(1):54-63, 2005.

8. George Jöngren, Mikael Skoglund, and Björn Ottersten. Combining beamforming and orthogonal space-time block coding. IEEE Transactions on Information Theory, 48(3):611-627, 2002.

9. Apinya Innok, Peerapong Uthansakul, and Monthippa Uthansakul. Angular beamforming technique for MIMO beamforming system. International Journal of Antennas and Propagation, 2012, 2012.

10. Siavash M Alamouti. A simple transmit diversity technique for wireless communications. IEEE Journal on Selected Areas in Communications, 16(8):1451-1458, 1998.

11. KOO Anoh, Raed A Abd-Alhameed, GN Okorafor, JM Noras, J Rodriguez, and SMR Jones. Performance evaluation of spatial modulation and QOSTBC for MIMO systems. EAI Endorsed Transactions on Mobile Communications and Applications, 15(6):e5, 2015.

12. Ke-Lin Du and Madisetti NS Swamy. Wireless communication systems: from $R F$ subsystems to $4 G$ enabling technologies. Cambridge University Press, 2010.

13. Andrea Goldsmith. Wireless communications. Cambridge university press, 2005.

14. John G Proakis and M Salehi. Digital communications. Fifth edition, 2008.

15. David J Love, Robert W Heath Jr, and Thomas Strohmer. Grassmannian beamforming for multiple-input multiple-output wireless systems. IEEE Transactions on Information Theory, 49(10):2735-2747, 2003.

16. KOO Anoh, SMR Jones, RAA Abd-Alhameed, TT Mapoka, GN Okorafor, and MJ Ngala. A simple space-time coding technique for wireless communication systems. In Internet Technologies and Applications (ITA), 2015, pages 405-410, 2015.

17. Unhee Park, Sooyoung Kim, Kwangjae Lim, and Jing Li. A novel QOSTBC scheme with linear decoding for three and four transmit antennas. IEEE Communications Letters, 12(12):868-870, 2008.

18. Van-Bien Pham, Bo-Yu Qi, Wei-Xing Sheng, and Mao Wang. An improved full rate full diversity qostbc with linear decoding in mimo systems. Wireless personal communications, 69(1):121-131, 2013.

19. Van-Bien Pham and Wei-Xing Sheng. No-zero-entry full diversity space-time block codes with linear receivers. annals of telecommunications-annales des télécommunications, 70(1-2):73-81, 2015. 